\title{
Variación morfológica de la lagartija partenogenética Aspidoscelis rodecki (Squamata: Teiidae): implicaciones evolutivas y de conservación
}

\author{
Sandra P. Elizalde-Rocha1, Fausto R. Méndez-de la Cruz², J. Fernando Méndez-Sánchez ${ }^{1}$, \\ Gisela Granados-González ${ }^{1}$ \& Oswaldo Hernández-Gallegos ${ }^{1}$ \\ 1. Centro de Investigación en Recursos Bióticos. Carretera Toluca-Ixtlahuaca Km 14.5. San Cayetano Toluca, Estado de \\ México.México; pandora_bio@yahoo.com.mx,fms@uaemex.mx, gis_gmx@yahoo.com.mx, ohg@uaemex.mx \\ 2. Departamento de Zoología, Instituto de Biología, Universidad Nacional Autónoma de México, A.P. 70-173, C.P. \\ 04510, 20, D.F. México; faustor@ibiologia.unam.mx
}

Recibido 15-I-2008. Corregido 30-VI-2008. Aceptado 31-VII-2008.

\begin{abstract}
Morphologic variation of the parthenogenetic lizard Aspidoscelis rodecki (Squamata: Teiidae): evolutionary and conservation implications. Post-formational divergence has been used for the recognition of new parthenogenetic species. Currently, the parthenogenetic lizard Aspidoscelis rodecki McCoy and Maslin 1962 is recognized as a single taxon that was derived from a single, parthenogenetically capable, hybrid. This lizard had been derived via hybridization between individuals of two gonochoristic species, Aspidoscelis angusticeps Cope 1878 and Aspidoscelis deppii Wiegmann 1834. The distribution of A. rodecki includes Isla Contoy and Isla Mujeres and the adjacent mainland of Quintana Roo, México. Previous studies have found post-formational divergence in genetic, chromatic and life-history characteristics among a continental population (Puerto Juárez) and an insular population (Isla Contoy). A meristic analysis was carried out to evaluate the morphological divergence among both populations of $A$. rodecki. We used 38 individuals from Puerto Juárez and 23 individuals from Isla Contoy. Nine meristic characters with discrimination value among species of the genus Aspidoscelis were used in both univariate (t-Student) and multivariate analyses (principal components and canonical variate analysis). According to both analyses, Puerto Juárez is meristically distinguishable from Isla Contoy. Both populations differ in five meristic characters and were a high correct classification in the canonical variate analysis: $97 \%$ of Puerto Juárez and $100 \%$ of Isla Contoy. A small sample from Isla Mujeres and a single specimen from Punta Sam (mainland) may represent different morphological groups. Due to the patterns of phenotypic variation, $A$. rodecki is considered as a single variable parthenogenetic species with high priority to conservation. The populations of $A$. rodecki have been extremely affected by the tourism developers. If the habitat of the parthenogenetic lizard (beach grasses) is allowed to stay, the expansion by the developers will not affect the survivorship of these populations. Nevertheless, the first sign of development is the total destruction of natural grasses that occurs on the beach, leaving only sand. There is a last chance to save the parthenogenetic lizard $A$. rodecki, but any effort will be useless without the support from the environmental authority of Mexico and cooperation from the developers. We suggest that Puerto Juárez and Isla Contoy receive separate management because they have unique portions of phenotypic variation of $A$. rodecki. The two lizard populations can be considered separate "Evolutionary Significant Units" (ESU). Rev. Biol. Trop. 56 (4): 1871-1881. Epub 2008 December 12 .
\end{abstract}

Key words: Squamata, Teiidae, Aspidoscelis rodecki, parthenogenesis, morphological variation.

De acuerdo a Flores-Villela (1993), en México existen diez especies partenogenéticas del género Aspidoscelis Reeder et al. 2002. Las tres especies formalmente nombradas de la Península de Yucatán (Aspidoscelis rodecki
McCoy y Maslin 1962, A. maslini Taylor y Cooley 1995 y A. cozumela Gadow 1906) forman parte del grupo A. cozumela (Wright 1993) y se consideraron dentro de una misma especie (Gadow 1906). Fritts (1969) elevó 
a $A$. rodecki a nivel de subespecie con base en escutelación y coloración. Esta especie se originó como híbrido entre un macho de $A$. deppii Wiegmann 1834 y una hembra de $A$. angusticeps Cope 1878 (Fritts 1969, Moritz et al. 1992, Taylor y Cooley 1995a, b, HernándezGallegos et al. 1998, Manríquez-Morán 2002, Hernández-Gallegos et al. 2003). Su distribución se restringe al noreste de Quintana Roo en la Península de Yucatán, México, en poblaciones continentales (Puerto Juárez, Punta Sam y Punta Nizuc) e insulares (Isla Contoy e Isla Mujeres) (Lee 1996).

McCoy y Maslin (1962), registraron que los individuos de las poblaciones de Puerto Juárez e Isla Mujeres no diferían en escutelación, patrón de coloración y talla. Sin embargo, un solo individuo de Isla Contoy presentó mayor número de escamas alrededor de la región media del cuerpo y los autores dudaron si dicha variación era individual o poblacional. Fritts (1969) registró diferencias en estas poblaciones en la región frontonasal-prefrontal. De acuerdo a Taylor y Cooley (1995a), $A$. rodecki presentó la menor variabilidad morfológica respecto de A. maslini y A. cozumela. Los autores no encontraron diferencias morfológicas entre la población Puerto Juárez e Isla Mujeres. Recientemente, Hernández-Gallegos et al. (2003) registraron variación cromática entre Puerto Juárez e Isla Contoy.

Las poblaciones de $A$. rodecki difieren escasamente a nivel genético. ManríquezMorán (2002) registró un solo cariotipo y dos clones (mediante el uso de genes mitocondriales) que corresponden a Puerto Juárez e Isla Contoy; la distancia genética entre ambas poblaciones, corregida con el modelo general de tiempo reversible fue mínima (0.0027). La histocompatibilidad dentro $(100 \%)$ y entre poblaciones $(97.9 \%)$ indicó que $A$. rodecki se originó de un solo híbrido partenogenético (Hernández-Gallegos et al. 2003).

A nivel ecológico existe cierta divergencia. Hernández-Gallegos (1998) analizó las poblaciones de Puerto Juárez e Isla Contoy y encontró diferencias en características reproductoras y de historia de vida, algunas de ellas de acuerdo a las predicciones teóricas de organismos insulares y continentales. El tamaño de nidada y madurez sexual fue mayor en Isla Contoy y el período reproductor fue ligeramente diferente entre poblaciones.

En Aspidoscelis actualmente se conocen ejemplos de divergencia, después de la formación, que en algunos casos ha sido suficiente para el reconocimiento de nuevas especies (para una revisión reciente ver ManríquezMorán 2007). La divergencia morfológica (Taylor y Cooley 1995a, Elizalde-Rocha 2007) y citogenética después de la formación en una hembra de A. maslini de Puerto Morelos, aparentemente originó A. cozumela (ManríquezMorán et al. 2000). La evidencia anterior y su aislamiento geográfico (Lee 1996, HernándezGallegos et al. 1998, Manríquez-Morán 2002) fueron elementos suficientes para dar identidad y elaborar una diagnosis cohesiva para asignarlas como especies diferentes (Taylor y Cooley 1995a, Manríquez-Morán et al. 2000, Taylor et al. 2005). De acuerdo al concepto evolutivo de especie, dicha identidad es una condición necesaria para el reconocimiento formal de un linaje partenogenético (Taylor et al. 2005). La diversidad fenotípica (adquirida después de la formación) en las diferentes clases de $A$. tesselata Say 1823 (Taylor et al. 2005) y en las diferentes poblaciones de $A$. maslini (ElizaldeRocha 2007) impiden identidad en alguna clase o población de estas dos especies partenogenéticas. En ausencia de identidad, se ha sugerido que ambas especies representan linajes partenogenéticos altamente variables (Taylor et al. 2005, Elizalde-Rocha 2007).

La lagartija partenogenética $A$. rodecki se encuentra en la Norma Oficial Mexicana (NOM-059-ECOL-2001) como especie sujeta a protección especial (Méndez de la Cruz et al. 2003) y existe una propuesta para su reclasificación a especie en peligro de extinción (Hernández-Gallegos 2004). La distribución restringida de $A$. rodecki en la costa noreste de Quintana Roo, su elevada dependencia por las playas, el constante y desmedido desarrollo urbano que elimina su hábitat, hacen de esta lagartija una especie severamente amenazada y 
prioritaria para la conservación (Méndez de la Cruz et al. 2003).

La evidencia disponible sugiere que $A$. rodecki presenta variación morfológica y de coloración entre las hembras de Puerto Juárez e Isla Contoy. Sin embargo, debido a la carencia de hembras de Isla Contoy (aparentemente diferentes sensu McCoy y Maslin 1962), su variación en escutelación no se había documentado. Por ello, el presente trabajo realiza una comparación de la escutelación de las hembras de la lagartija partenogenética $A$. rodecki de Puerto Juárez e Isla Contoy, Quintana Roo, México. El objetivo fue valorar la divergencia morfológica entre ambas poblaciones, así como las implicaciones evolutivas y en la conservación de esta especie.

\section{MATERIAL Y MÉTODOS}

Poblaciones involucradas: el análisis morfológico se realizó con las hembras de Puerto Juárez $(n=38)$ e Isla Contoy $(n=23)$. Adicionalmente, se incluyó una pequeña muestra de Isla Mujeres $(n=2)$ y un individuo de Punta Sam. Los cuatro sitios se localizan al noreste de Quintana Roo, en la Península de Yucatán, México (ver Hernández-Gallegos et al. 2003). Los ejemplares se depositaron en la Colección Nacional de Anfibios y Reptiles (CNAR 21657-21720).

Características analizadas: a cada espécimen se le realizó un conteo de nueve características de escutelación, que han demostrado discriminación entre las especies del género Aspidoscelis (Apéndice 1). Estas características se emplearon previamente en el complejo A. cozumela (Taylor y Cooley 1995a). Cabe señalar que se mantuvieron las mismas siglas utilizadas por los autores. Los conteos de escamas se llevaron a cabo por una sola persona (SPE-R) e incluyen la suma de ambos lados de cada individuo.

Análisis estadísticos: para evaluar las diferencias entre las medias de las nueve características de escutelación, se realizó un análisis de $\mathrm{t}$ de Student. Debido al bajo número de muestra en Isla Mujeres y Punta Sam, los individuos provenientes de ambos sitios no se incluyeron en este análisis.

Para explorar los caracteres merísticos que podrían definir las poblaciones, se realizó un análisis de componentes principales (ACP) basado en una matriz de correlación. Para contrastar la hipótesis de que al menos una población es diferente en las variables canónicas compuestas de la contribución parcial de los caracteres merísticos originales, se aplicó un análisis de variación canónica (AVC). Para evaluar el parecido entre las poblaciones de Puerto Juárez e Isla Contoy, mediante igualdad de grupos, se obtuvo una tabla de clasificación. Los análisis univariados y multivariados se realizaron en Statgraphics Plus 5.0. Se usó un alfa de 0.05 .

\section{RESULTADOS}

Los análisis univariados incluyeron sólo las poblaciones más numerosas de $A$. rodecki: Puerto Juárez e Isla Contoy. Los análisis multivariados incluyeron los cuatro sitios de recolecta: Puerto Juárez, Isla Contoy, Isla Mujeres y Punta Sam.

Análisis univariados: de las nueve características de escutelación analizadas, se obtuvo la media y el error estándar de Puerto Juárez e Isla Contoy (Cuadro 1). De acuerdo a los análisis de $\mathrm{t}$ de Student, ambas poblaciones se encuentran separadas por cinco de las nueve características de escutelación: LSG-2, PSC, GS, FP y SDL-1 (todos los valores de $\mathrm{p}<0.05$ ). Ambas poblaciones no difieren en: FRP, COS y GAB (todos los valores de $\mathrm{p} \geq 0.05$ ). No se realizó la comparación de las escamas SO, debido a que en Puerto Juárez las escamas mantuvieron un número constante.

Análisis multivariados: en el ACP se observó un patrón de variación paralelo al componente principal 1 (CP1), donde Isla Contoy y Puerto Juárez son los extremos y Punta Sam e Isla Mujeres son intermedias. Los caracteres que contribuyeron con más peso en este patrón son COS, FP y LGS-2 hacia Isla Contoy y PSC para Puerto Juárez (Fig. 1A, Cuadro 2). También se observó una variación al interior 


\section{CUADRO 1}

Medias, error estándar y comparación de medias ( $t$ de Student), de cada uno de los caracteres de escutelación utilizados en dos poblaciones de A. rodecki

TABLE 1

Means, standard error and mean comparison (t-Student) of each scutellation character determined in two A. rodecki populations

$\begin{array}{lcccc}\text { Caracter } & \text { Puerto Juárez } \mathrm{n}=38 & \text { Isla Contoy } \mathrm{n}=23 & \mathrm{t} & \mathrm{p} \\ \text { FRP } & 2.1 \pm 0.1 & 2.3 \pm 0.1 & 1.76 & 0.09 \\ \text { COS } & 28.5 \pm 0.3 & 32.8 \pm 0.5 & 5.42 & 0.05 \\ \text { LSG-2 } & 34.8 \pm 0.4 & 39.5 \pm 0.5 & 7.39 & <0.0001 \\ \text { PSC } & 22.3 \pm 0.2 & 21.2 \pm 0.4 & -2.34 & 0.02 \\ \text { SO } & 8.0 \text { valor constante } & 8.0 \pm 0.5 & \text { no aplica } & \text { no aplica } \\ \text { GS } & 26.8 \pm 0.4 & 32.4 \pm 0.5 & 8.91 & <0.0001 \\ \text { FP } & 33.0 \pm 0.2 & 36.0 \pm 0.3 & 8.18 & <0.0001 \\ \text { SDL-1 } & 31.3 \pm 0.2 & 33.0 \pm 0.3 & 4.46 & 0.0001 \\ \text { GAB } & 103.3 \pm 0.5 & 104.9 \pm 0.8 & 1.73 & 0.09\end{array}$

n: tamaño de muestra. Las abreviaciones en los caracteres corresponden a las listadas en el Apéndice 1.

n: sample size. To abbreviations of all listed characters see Apendix 1.

\section{CUADRO 2}

Cargas para los primeros tres componentes principales derivados de cuatro localidades de A. rodecki (Puerto Juárez, Isla Contoy, Isla Mujeres y Punta Sam)

TABLE 2

Principal component loadings for the A. rodecki localities (Puerto Juárez, Isla Contoy, Isla Mujeres and Punta Sam)

$\begin{array}{lccc}\text { Caracter } & \text { Componente } 1 & \text { Componente } 2 & \text { Componente } 3 \\ \text { FRP } & 0,139909 & 0,586957 & 0,288556 \\ \text { GAB } & 0,222852 & 0,123448 & -0,00590853 \\ \text { COS } & 0,464238 & -0,168503 & 0,246561 \\ \text { LSG-2 } & 0,42312 & -0,179871 & 0,362444 \\ \text { FP } & 0,446042 & -0,052285 & -0,0237144 \\ \text { SDL-1 } & 0,38225 & -0,00166165 & -0,34153 \\ \text { PSC } & -0,155675 & -0,315707 & 0,726984 \\ \text { SO } & 0,0470939 & 0,689974 & 0,233045 \\ \text { GS } & 0,405825 & -0,0319935 & -0,156594 \\ \% \text { de varianza explicada } & 34.24 & 15.5 & 13.25\end{array}$

Las abreviaciones en los caracteres corresponden a las listadas en el Apéndice 1.

To abbreviations of all listed characters see Apendix 1. 
A

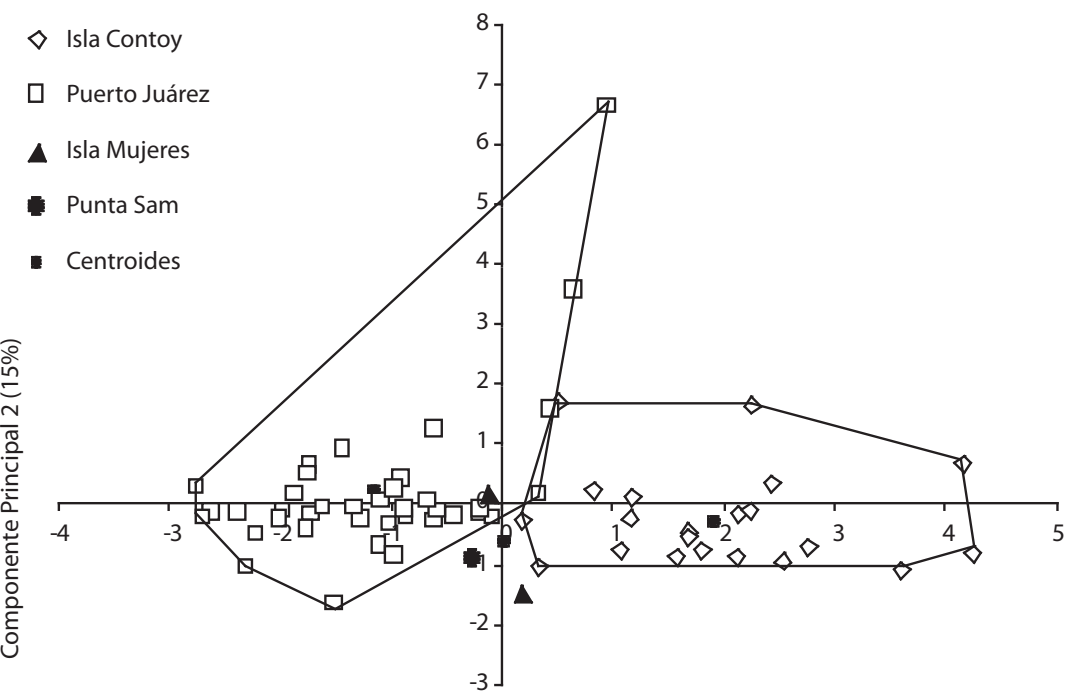

Componente Principal 1 (34\%)

B
$\diamond$ Isla Contoy
प Puerto Juárez
$\Delta$ Isla Mujeres
- Punta Sam
- Centroides

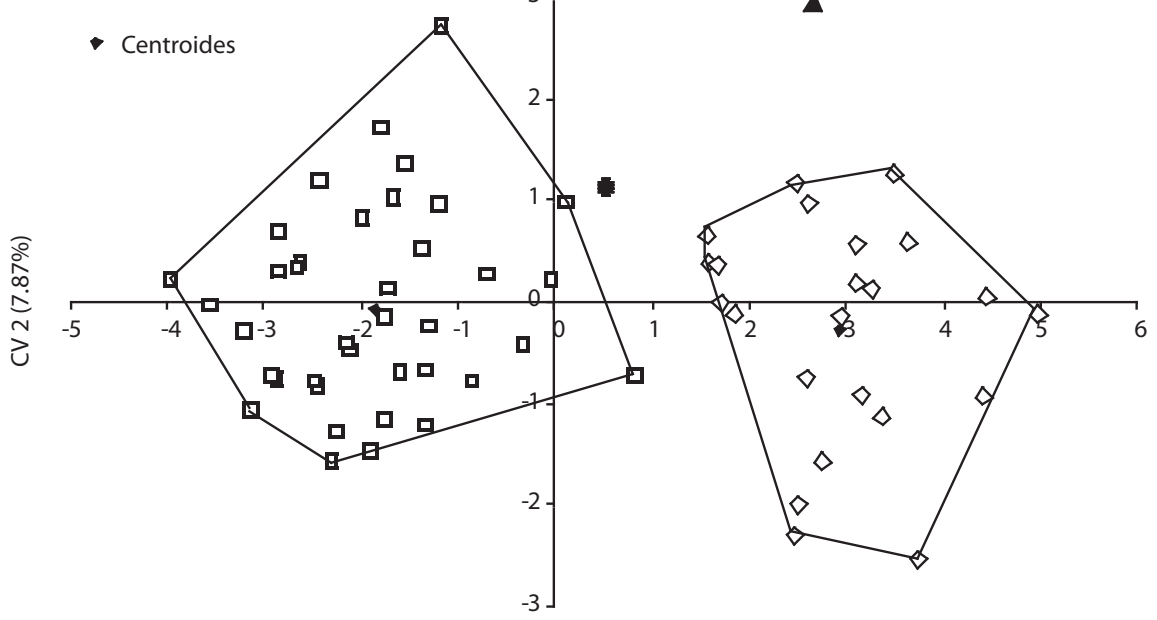

CV 1 (91.14\%)

Fig. 1. Variación morfológica multivariada entre cuatro poblaciones de A. rodecki. A. Análisis de componentes principales. B. Análisis de variación canónica. El porcentaje de los ejes se refiere a la varianza explicada y los polígonos delimitan a las poblaciones de Puerto Juárez e Isla Contoy.

Fig. 1. Multivariate morphological variation among four populations of $A$. rodecki. A. Principal component analysis. B. Canonical variates analysis. The axis percentage refers to the explained variance and the polygons delimit Puerto Juárez and Isla Contoy populations. 
de la población de Puerto Juárez paralela al componente principal 2 (CP2) y éste se asocia básicamente con los caracteres GS y FRP.

El AVC indicó que en la primera variable canónica se separan las dos poblaciones de interés (Puerto Juárez e Isla Contoy) (Fig. 1B, Cuadro 3) (lambda de Wilks=0.097, gl=3, 52, $\mathrm{p}<0.0001)$. Sobre esta variable, las características de escutelación con un mayor coeficiente estandarizado de discriminación para separar a la población de Isla Contoy fueron FP (0.783) y GS (0.734) y a la de Puerto Juárez fue SO (-0.413). En estos análisis, se observó que los individuos de Isla Mujeres y Punta Sam podrían representar entidades de escutelación diferentes respecto a Puerto Juárez e Isla Contoy, sin embargo, estos resultados no pueden ser concluyentes al tener una muestra pequeña.

Los porcentajes de clasificación indicaron una asignación correcta del 97.37\% (37 de 38) para Puerto Juárez y del 100\% (23 de 23) para Isla Contoy. La clasificación total promedio fue del 98.68\% (Cuadro 4).

\section{DISCUSIÓN}

Variación morfológica en $A$. rodecki: a pesar de su modo de reproducción clonal, la variación fenotípica se ha documentado en varias especies partenogenéticas (Parker et al. 1989, Walker et al. 1997, Hernández-Gallegos
CUADRO 3

Valores del análisis de variación canónica realizado para buscar las diferencias entre las cuatro poblaciones de $\mathrm{A}$. rodecki

TABLE 3

Standardized coefficients and canonical variate analysis values of $\mathrm{A}$. rodecki populations

$\begin{array}{lccc}\text { Caracter } & \text { CV1 } & \text { CV2 } & \text { CV3 } \\ \text { FRP } & 0.054 & 0.439 & -0.634 \\ \text { GAB } & -0.113 & -0.454 & -0.664 \\ \text { COS } & 0.255 & -0.101 & 0.333 \\ \text { LSG-2 } & 0.404 & 0.030 & 0.476 \\ \text { FP } & 0.783 & 0.405 & -0.418 \\ \text { SDL-1 } & -0.121 & -0.241 & -0.111 \\ \text { PSC } & -0.010 & 0.783 & -0.315 \\ \text { SO } & -0.413 & -0.105 & 0.354 \\ \text { GS } & 0.734 & -0.148 & -0.0237 \\ \text { \% de varianza explicada } & 91.14 & 7.87 & 0.99 \\ \text { Correlación canónica } & 0.92 & 0.56 & 0.23 \\ \text { Lambda de Wilks } & 0.097 & 0.63 & 0.94 \\ \text { p } & <0.0001 & 0.06 & 0.85\end{array}$

CV1, CV2, CV3: variables canónicas derivadas del análisis de variación canónica. Las abreviaciones en los caracteres corresponden a las listadas en el Apéndice 1.

CV1, CV2, CV3: canonical variate from canonical variate analysis. To abbreviations of all listed characters see Apendix 1.

CUADRO 4

Porcentaje de clasificación para poblaciones de A. rodecki, basado en el análisis de variación canónica. Se muestra el número de individuos y entre paréntesis los porcentajes de clasificación correcta

TABLE 4

Canonical variates classification percentage of A. rodecki populations. The number of individuals and percentages (in parenthesis) are showed

Población actual

$\begin{array}{ll}\text { Puerto Juárez } & \\ \text { Isla Contoy } & \\ \text { n: tamaño de muestra. } \\ \text { n: sample size. }\end{array}$

Población clasificada por el análisis de variación canónica

$\begin{array}{cc}\text { Puerto Juárez } & \text { Isla Contoy } \\ 37(97.37) & 1(2.63) \\ 0(0.0) & 23(100)\end{array}$


et al. 1998, Elizalde-Rocha 2007). En este estudio, los análisis univariados y multivariados indicaron que Puerto Juárez es merísticamente distinguible de Isla Contoy. Es decir, existe una evidente variación de escutelación en la lagartija partenogenética $A$. rodecki en Quintana Roo, México. La marcada diferenciación entre poblaciones se apoya en el hecho de que ambas varían entre sí en el $55.5 \%$ de las características analizadas (LSG-2, PSC, GS, FP y SDL-1) $\mathrm{y}$ presentaron un porcentaje de clasificación muy elevado. Estos resultados concuerdan con la evidencia de que ambas poblaciones diferían en su coloración, líneas paravertebrales (Hernández-Gallegos et al. 2003) y número de escamas alrededor de la región media del cuerpo (McCoy y Maslin 1962).

La evidencia actual sugiere que la variación morfológica (escutelación y cromática) de $A$. rodecki, no es individual sino poblacional. Con ello, se clarifica la duda expresada previamente por McCoy y Maslin (1962). Los resultados de otras localidades de $A$. rodecki (i. e., Isla Mujeres y Punta Sam; para una variación nula de la población de Isla Mujeres ver McCoy y Maslin 1962, Taylor y Cooley 1995a), indican que posiblemente también han divergido morfológicamente, sin embargo, debido a su bajo número no es posible ser concluyente.

Los estudios realizados indican que la divergencia genética, morfológica y ecológica que presenta $A$. rodecki es posterior a su formación, ya que aparentemente esta especie se originó de una sola hembra (Hernández-Gallegos et al. 2003). Ejemplos de divergencia, después de su formación en linajes partenogenéticos, lo constituyen A. maslini (Taylor y Cooler 1995a, Hernández-Gallegos et al. 1998, ManríquezMorán 2002, Elizalde-Rocha 2007) y A. tesselata (Taylor et al. 2005).

Actualmente se desconoce el factor que promueve la variación morfológica en $A$. rodec$k i$. Sin embargo, estudios previos sugieren que la variación en escutelación puede ser producto de diferencias ambientales durante el desarrollo embrionario (Fox et al. 1961, Lourdais et al. 2004) ó que es una fluctuación temporal (Vanzolini 2001). En la variación morfológica de $A$. rodecki, no se puede descartar la influencia genética.

Implicaciones evolutivas: estudios previos sugirieron que las especies clonales representaban fines evolutivos (Vrijenhoek 1989). Sin embargo, diferentes especies partenogenéticas, que incluyen todas las del género Aspidoscelis, presentan diversos grados de variación fenotípica (Dessauer y Cole 1989, Murphy et al. 1997, Manríquez-Morán 2007). Los métodos para evaluar dicha variación y los conceptos filosóficos pueden afectar la taxonomía de las especies clonales (Taylor et al. 2005, Manríquez-Morán 2007), la cual generalmente es controversial (Cole 1985, Frost y Wright 1988, Echelle 1990). Por ejemplo, de acuerdo a Coyne y Orr (2004), en un escenario reduccionista, en una especie clonal cada individuo con un cambio genético, puede ser considerado una especie diferente. Por el contrario, H. L. Taylor (comentario personal), considera que ciertas mutaciones pueden afectar la expresión de caracteres morfológicos, de modo que diferentes clones pueden establecerse en diversas localidades. Evidencia reciente sugiere que la divergencia fenotípica en linajes partenogenéticos, después de su formación, puede constituir una oportunidad para la especiación. El reconocimiento de dicha divergencia, se acompañó de un cambio en el pensamiento de lo que constituía una especie partenogenética (para una revisión reciente ver Taylor et al. $2005 \mathrm{y}$ Manríquez-Morán 2007).

Tomando en cuenta la evidencia disponible en $A$. rodecki, Puerto Juárez e Isla Contoy difieren en su morfología (McCoy y Maslin 1962, Hernández-Gallegos et al. 2003, este estudio), a nivel genético (Manríquez-Morán 2002, Hernández-Gallegos et al. 2003), en algunas características de historia de vida (HernándezGallegos 1998) y existen barreras geográficas entre las poblaciones (Lee 1996). Sin embargo, ambas poblaciones presentan el mismo cariotipo (Manríquez-Morán 2002). La evidencia disponible no permite elaborar una diagnosis cohesiva que separe a Puerto Juárez de Isla Contoy. Por lo anterior, se sugiere que $A$. rodec$k i$, es una lagartija partenogenética variable y 
que experimenta una situación similar a otras especies partenogenéticas como A. maslini (Elizalde-Rocha 2007) y A. tesselata (Taylor et al. 2005). De acuerdo al concepto evolutivo de especie, la identidad es una condición necesaria para el reconocimiento formal de un linaje partenogenético (Taylor et al. 2005).

Implicaciones en conservación: diferentes razones existen para conservar las especies clonales de vertebrados (Kraus 1995). La lagartija partenogenética $A$. rodecki es endémica de México y está incluida en la lista de especies en riesgo como especie sujeta a protección especial (Méndez de la Cruz et al. 2003). Su distribución se restringe al noreste de Quintana Roo en la Península de Yucatán, México (Lee 1996, Méndez de la Cruz et al. 2003). Estudios recientes indican que su distribución potencial es muy similar a su distribución conocida; lo que indica que los sitios donde habita $A$. rodec$k i$, deben considerarse como prioritarios para la conservación (Ballesteros-Barrera et al. 2004).

Los registros más recientes indican que A. rodecki estaba presente en Isla Contoy, Isla Mujeres, Puerto Juárez y Punta Sam; desafortunadamente, debido a su elevada dependencia por las playas y su vegetación, algunas poblaciones fueron extirpadas (Punta Nizuc) o disminuidas (Isla Mujeres) localmente por desarrollos turísticos (Méndez de la Cruz et al. 2003). La población de Isla Mujeres fue severamente afectada desde hace dos décadas, pero alrededor del aeropuerto existía hábitat disponible y algunos individuos de $A$. rodec$k i$ persistían. Sin embargo, una evaluación en mayo de 2008, indicó que el hábitat y las lagartijas fueron completamente extirpados de la isla. Durante dicha evaluación, una situación similar ocurrió en Puerto Juárez, donde un complejo turístico eliminó totalmente el hábitat y las lagartijas de la localidad. Con estos hallazgos, Isla Contoy podría representar el único sitio con nula afectación por los desarrollos turísticos (Méndez de la Cruz et al. 2003), aunque su densidad es relativamente baja comparada con otras poblaciones (McCoy y Maslin 1962, Hernández-Gallegos y Méndez-de la Cruz 2000). Por lo anterior, la lagartija partenogenética $A$. rodecki resulta ser una especie severamente amenazada y debe ser prioritaria para la conservación.

Debido a su evidente divergencia genética (a pesar de su modo de reproducción clonal: ausencia de intercambio genético; Manríquez-Morán 2002, Hernández-Gallegos et al. 2003), morfológica (McCoy y Maslin 1962, Hernández-Gallegos et al. 2003, este estudio) y ecológica (Hernández-Gallegos 1998), Puerto Juárez e Isla Contoy deben de tratarse como unidades independientes para su manejo y conservación. Se propone que cada población constituya una "Unidad Evolutiva Significativa" (ESU; Crandall et al. 2000). Lo anterior, en virtud de que cada una posee una porción única de la variación total de $A$. rodec$k i$. Dicha variación puede ser una oportunidad para la especiación, como sucedió en A. maslini (Taylor y Cooley 1995a, Manríquez-Morán et al. 2000).

Debido a la disminución significativa en la distribución de $A$. rodecki respecto a su distribución histórica (Lee 1996), se deben realizar esfuerzos conjuntos entre las autoridades ambientales en México y los desarrollos turísticos. De acuerdo a la experiencia en la Península de Yucatán, es posible la combinación de dichos desarrollos y la conservación de flora y fauna en las costas (HernándezGallegos 2004).

El papel de los hábitats insulares: se ha sugerido (Lynch y Gabriel 1983) que las especies partenogenéticas tienen tasas de evolución fenotípica similar o mayor que las especies gonocóricas (con dos sexos). Aparentemente, la variación fenotípica de las especies clonales está en relación a su ecología, amplitud de su distribución y a la edad de su formación (Dessauer y Cole 1989, Parker et al. 1989, Hernández-Gallegos et al. 1998, 2003). Existen diferentes factores que influyen en dicha variación, entre ellos mutación, recombinación y/o múltiples orígenes (Murphy et al. 1997). Es posible que la variación fenotípica se deba a cambios genéticos y/o influencias ambientales.

Debido a su modo de reproducción clonal (identidad genética), las especies 
partenogenéticas representan laboratorios naturales, un ejemplo de ello lo constituye $A$. rodecki y las otras especies del complejo $A$. cozumela. En un estudio reciente, ElizaldeRocha (2007) sugirió que, debido a un rápido cambio adaptativo, los ambientes insulares favorecen la diferenciación de los individuos partenogenéticos. En este estudio, se comentó que las poblaciones insulares de las diferentes especies del complejo A. cozumela, sufren modificaciones fenotípicas en tres aspectos: 1) genético, 2) cariológico y 3) morfológico. Dicha evidencia sugiere que los ambientes insulares, por medio de condiciones de selección diferentes, pueden acelerar la divergencia entre poblaciones de especies partenogenéticas (Hernández-Gallegos 2004), aun en taxones con distribución restringida como es el caso de A. rodecki.

\section{AGRADECIMIENTOS}

A los proyectos PROMEP Convenio 103.5/04/2644, UAEMéx 2295/2006 y 2620/2008 por el apoyo financiero para Oswaldo Hernández-Gallegos durante la redacción de este trabajo. A PAPIIT por el apoyo financiero para realizar el trabajo de campo. A F. Rodríguez por su ayuda en el campo. A dos revisores anónimos por comentarios que mejoraron sustancialmente este trabajo.

\section{RESUMEN}

La divergencia post-formación se ha utilizado para el reconocimiento de nuevas especies partenogenéticas. Actualmente, la lagartija partenogenética Aspidoscelis rodecki McCoy y Maslin 1962 es reconocida como una sola especie, que se originó de un híbrido partenogenético. Estudios previos han encontrado divergencia genética, en coloración y en características de historia de vida entre una población continental (Puerto Juárez) y una insular (Isla Contoy) en Quintana Roo, México. Se llevó a cabo un análisis merístico para evaluar la divergencia entre ambas poblaciones de $A$. rodecki. Se utilizaron 38 individuos de Puerto Juárez y 23 individuos de Isla Contoy. Se usaron nueve características merísticas y se realizaron análisis univariados (t de Student) y multivariados (análisis de componentes principales y análisis de variación canónica). De acuerdo a ambos análisis, Puerto Juárez es merísticamente distinguible de Isla Contoy. Ambas poblaciones difieren en cinco características merísticas y presentaron un alto porcentaje de clasificación en el análisis de variación canónica: $97 \%$ para Puerto Juárez y 100\% para Isla Contoy. Una pequeña muestra de Isla Mujeres y un solo individuo de Punta Sam (continente) pueden representar otros grupos morfológicos. Con base en los patrones de variación fenotípica, A. rodecki es considerada como una sola especie partenogenética variable y prioritaria para la conservación. Debido a que cada población contiene una porción única de la variación de $A$. rodecki, Puerto Juárez e Isla Contoy merecen un manejo separado y se sugiere que cada una constituya una "Unidad Evolutiva Significativa" (ESU).

Palabras clave: Squamata, Teiidae, Aspidoscelis rodecki, partenogénesis, variación morfológica.

\section{REFERENCIAS}

Ballesteros-Barrera, C., O. Hernández-Gallegos, C. González-Salazar \& E. Martínez-Meyer. 2004. Modelado del nicho ecológico para especies con distribución restringida: implicaciones para su conservación. Memorias de la VIII Reunión Nacional de Herpetología, Tabasco, México.

Cole, C.J. 1985. Taxonomy of parthenogenetic species of hybrid origin. Syst. Zool. 34: 359-363.

Cole, C.J., H.C. Dessauer \& G.F. Barrowclough. 1988. Hybrid origin of a unisexual species of whiptail lizard, Cnemidophorus neomexicanus, in western North America: new evidence and a review. Amer. Mus. Novit. 2905: 1-38.

Coyne, J.A. \& H.A. Orr. 2004. Speciation. Sinauer, Sunderland, Massachusetts, EEUU.

Crandall, K.A., O.R.P. Bininda-Emonds, G.M. Mace \& R.K. Wayne. 2000. Considering evolutionary processes in conservation biology. Trends Ecol. Evol. 15: 290-295.

Dessauer, H.C. \& C.J. Cole. 1989. Diversity between and within nominal forms of unisexual teiid lizards, p. 49-71. In R.M. Dawley \& J.P. Bogart (eds.). Evolution and Ecology of Unisexual Vertebrates. Bulletin 466. New York State Museum, Albany, Nueva York, EEUU.

Echelle, A.E. 1990. In defense of the phylogenetic species concept and the ontological status of hybridogenetic taxa. Herpetologica 46: 109-113.

Elizalde-Rocha, S.P. 2007. Evolución y sistemática de las lagartijas partenogenéticas del género Aspidoscelis (Squamata: Teiidae) de la Península de Yucatán. Tesis 
de Licenciatura, Facultad de Ciencias, Universidad Autónoma del Estado de México, Toluca, Estado de México, México.

Flores-Villela, O. 1993. Herpetofauna Mexicana. Carnegie Museum of Natural History. Pittsburgh, EEUU.

Fox, W., C. Gordon \& M.H. Fox. 1961. Morphological effects of low temperatures during embryonic development of the garter snake, Thamnophis elegans. Zoologica 46: 57-71.

Fritts, T.H. 1969. The systematics of the parthenogenetic lizards of the Cnemidophorus cozumela complex. Copeia 1969: 519-535.

Frost, D.R. \& J.W. Wright. 1988. The taxonomy of uniparental species, with special reference to parthenogenetic Cnemidophorus (Squamata: Teiidae). Syst. Zool. 37: 200-209.

Gadow, H. 1906. A contribution to the study of evolution based upon the Mexican species of Cnemidophorus. Proc. Zool. Soc. London 1: 277-375.

Hernández-Gallegos, O. 1998. Histocompatibilidad y ciclo reproductor en dos poblaciones de la lagartija partenogenética Cnemidophorus rodecki, en el Edo. de Quintana Roo. Tesis de Maestría, Facultad de Ciencias, Universidad Nacional Autónoma de México, Distrito Federal, México.

Hernández-Gallegos, O., N. Manríquez-Morán, F.R. Méndez, M. Villagrán \& O. Cuellar. 1998. Histocompatibility in parthenogenetic lizards of the Cnemidophorus cozumela complex from the Yucatán Peninsula of Mexico. Biogeographica 74: 117-124.

Hernández-Gallegos, O. \& F.R. Méndez-de la Cruz. 2000. Cnemidophorus rodecki: a seriously threatened species at the Yucatán Peninsula. Memorias de la $80^{\text {th }}$ Annual Meeting American Society of Ichtiologists and Herpetologists, La Paz, Baja California Sur, México.

Hernández-Gallegos, O., F.R. Méndez, M. Villagrán-Santa Cruz \& O. Cuellar. 2003. Genetic homogeneity between populations of Aspidoscelis rodecki, a parthenogenetic lizard from the Yucatán Peninsula. J. Herpetol. 37: 527-532.

Hernández-Gallegos, O. 2004. Demografía de los lacertilios del complejo Aspidoscelis (Cnemidophorus) cozumela (Sauria: Teiidae) y sus especies parentales, en la Península de Yucatán, México. Tesis de Doctorado, Universidad Nacional Autónoma de México, Distrito Federal, México.
Kraus, F. 1995. The conservation of unisexual vertebrate populations. Conserv. Biol. 9: 956-959.

Lee, J.C. 1996. The amphibians and reptiles of the Yucatán Peninsula. Cornell University, Ithaca, Nueva York, EEUU.

Lourdais, O., R. Shine, X. Bonnet, M. Guillon \& G. Naulleau. 2004. Climate affects embryonic development in a viviparous snake, Vipera aspis. Oikos 104: 551-560.

Lowe, C.H. \& R.G. Zweifel. 1952. A new species of whiptail lizard (genus Cnemidophorus) from New Mexico. Bull. Chicago Acad. Sci. 9: 229-247.

Lynch, M. \& W. Gabriel. 1983. Phenotypic evolution and parthenogenesis. Am. Nat. 122: 745-764.

Manríquez-Morán, N.L., M. Villagrán-Santa Cruz \& F.R. Méndez-de la Cruz. 2000. Origin and evolution of the parthenogenetic lizards, Cnemidophorus maslini and C. cozumela. J. Herpetol. 34: 634-637.

Manríquez-Morán, N.L. 2002. Origen y diversidad clonal en las especies de lagartijas partenogenéticas del complejo Cnemidophorus cozumela (Reptilia: Teiidae). Tesis de Doctorado, Universidad Nacional Autónoma de México, Distrito Federal, México.

Manríquez-Morán, N.L. 2007. Diversidad clonal en los lacertilios unisexuales del género Aspidoscelis. Bol. Soc. Herpetol. Mex. 15: 1-12.

McCoy, C.J. \& T.P. Maslin. 1962. A review of the teiid lizard Cnemidophorus cozumelus and the recognition of a new race, Cnemidophorus cozumelus rodecki. Copeia 1962: 620-627.

Méndez de la Cruz, F.R., O. Hernández-Gallegos \& F. Rodríguez-Romero. 2003. Cnemidophorus rodecki. Elaboración de fichas de 5 especies de lacertilios: PROY-NOM-059-ECOL-2000. Instituto de Biología, Universidad Nacional Autónoma de México. Bases de datos SNIB-CONABIO. Proyecto W033. Distrito Federal, México.

Moritz, C., J.W. Wright, V. Singh \& W.M. Brown. 1992. Mitocondrial DNA analyses and the origin and relative age of the parthenogenetic Cnemidophorus. V. The cozumela species group. Herpetologica 48: 417-424.

Murphy, R.W., I.S. Darevsky, R.D. MacCulloch, J. Fu, L.A. Kupriyanova, D.E. Upton \& F. Danielyan. 1997. Old age, multiple formations or genetic plasticity? Clonal diversity in a parthenogenetic Caucasian rock lizard, Lacerta dahli. Genetica 101: 125-130. 
Parker, E.D., J.M. Walker \& M.A. Paulissen. 1989. Clonal diversity in Cnemidophorus: ecological and morphological consequences, p. 72-86. In R.M. Dawley \& J.P. Bogart (eds.). Evolution and Ecology of Unisexual Vertebrates. Bulletin 466. New York State Museum, Albany, Nueva York, EEUU.

Serena, M. 1984. Distribution and habitats of parthenogenetic and sexual Cnemidophorus lemniscatus (Sauria: Teiidae) in Surinam. Copeia 1984: 713-719.

Taylor, H.L. \& C.R. Cooley. 1995a. A multivariate analysis of morphological variation among parthenogenetic teiid lizards of the Cnemidophorus cozumela complex. Herpetologica 51: 67-76.

Taylor, H.L. \& C.R. Cooley. 1995b. Patterns of meristic variation among parthenogenetic teiid lizards (genus Cnemidophorus) of the Yucatán Peninsula and their progenitor species, $C$. angusticeps and $C$. deppii. J. Herpetol. 29: 583-592.

Taylor, H.L., J.M. Walker, J.E. Cordes \& G.J. Manning. 2005. Application of the evolutionary species concept to parthenogenetic entities: comparison of postformational divergence in two clones of Aspidoscelis tesselata and between Aspidoscelis cozumela and Aspidoscelis maslini (Squamata: Teiidae). J. Herpetol. 39: 266-277.

Vanzolini, P.E. 2001. Temporal fluctuations in scale and body proportions of Amazonian riparian lizards (Cnemidophorus, Sauria, Teiidae). Amazoniana 16: 539-563.
Vrijenhoek, R.C. 1989. Genetic and ecological constraints on the origins and establishment of unisexual vertebrates, p. 24-31. In R.M. Dawley \& J.P. Bogart (eds.). Evolution and Ecology of Unisexual Vertebrates. Bulletin 466. New York State Museum, Albany, Nueva York, EEUU.

Walker, J.M., H.L. Taylor \& T.P. Maslin. 1966. Evidence for specific recognition of the San Esteban whiptail lizard (Cnemidophorus estebanensis). Copeia 1966: 498-505.

Walker, J.M., J.E. Cordes \& H.L. Taylor. 1997. Parthenogenetic Cnemidophorus tesselatus complex (Sauria: Teiidae): a neotype for diploid C. tesselatus (Say, 1823), redescription of the taxon, and description of a new triploid species. Herpetologica 53: 233-259.

Wright, J.W. 1966. Variation in two sympatric whiptail lizards (Cnemidophorus inornatus and C. velox) in New Mexico. Southwest. Nat. 11: 54-71.

Wright, J.W. \& C.H. Lowe. 1967. Hibridization in nature between parthenogenetic and bisexual species of whiptail lizards (genus Cnemidophorus). Amer. Mus. Novit. 2286: 1-36.

Wright, J.W. 1993. Evolution of whiptail lizards (Genus Cnemidophorus), p. 27-82. In J.W. Wright \& L.J. Vitt (eds.). Biology of Whiptail Lizards (Genus Cnemidophorus). Oklahoma Museum of Natural History, Norman, Oklahoma, EEUU.

\section{APÉNDICE 1}

\section{Caracteres de escutelación utilizados, basados en Taylor y Cooley (1995a, b)}

\section{APENDIX 1}

\section{Meristic characters according to Taylor and Cooley (1995a, b)}

FRP-Número total de escamas frontoparietales (Fritts 1969); COS-Número total de escamas circumorbitales en ambos lados de la cabeza (Wright y Lowe 1967); LSG-2-Número total de gránulos supraoculares en ambos lados de la cabeza (el punto de referencia estándar de inicio de las escamas LSG-2, ha sido la línea de sutura entre la tercera y la cuarta SO: Walker et al. 1966); PSC-Suma total de escamas alrededor del perímetro de las escamas parietales e interparietales (J. M. Walker, datos no publicados); SO-Número total de escamas supraoculares; GS-Número de escamas gulares en el perímetro interno de los escudos de la barbilla (Cole et al. 1988, Serena 1984); FP-Número total de poros femorales; SDL-1-Número de laminas subdigitales en el cuarto dígito de la extremidad trasera (la extremidad izquierda fue elegida, si esta está intacta); GAB-Número de escamas dorsales alrededor de la región media del cuerpo (Lowe y Zweifel 1952) con la cuenta estandarizada en la décimo quinta fila ventral (Wright 1966). 
